\title{
Relative abundance and habitat selection of the montane guinea pig Cavia tschudii in a wetland at coastal desert with comments on its predators
}

\author{
Manuel Quispe-López ${ }^{1 *}$, Sue Barreda', Diego Marcelo-Carranza ${ }^{2}$, Victor Pacheco², Héctor Aponte", and Damaso W. Ramirez ${ }^{4,5}$ \\ ${ }^{1}$ División de Mastozoología, Centro de Ornitología y Biodiversidad (CORBIDI). Santa Rita 105 Dpto. 202, CP. 15023, Lima. Lima, \\ Perú. Email: manuel.artq@gmail.com (MQ-L); valerysue.bo@gmail.com (SB). \\ ${ }^{2}$ Departamento de Mastozoología, Museo de Historia Natural, Universidad Nacional Mayor de San Marcos. Av. Arenales 1256, CP. \\ 15072, Lima. Lima, Perú. Email: diego.marcelo@unmsm.edu.pe (DM-C); vpachecot@unmsm.edu.pe (VP). \\ ${ }^{3}$ Carrera de Biología Marina, Facultad de Ciencias Veterinarias y Biológicas, Universidad Científica del Sur. Panamericana Sur Km. \\ 19, CP. 15067, Lima. Lima, Perú. Email: haponte@cientifica.edu.pe (HA) \\ ${ }^{4}$ Dirección de Cursos Básicos, Universidad Científica del Sur. Panamericana Sur Km. 19, CP. 15067, Lima. Lima, Perú. Email: \\ dramirezh@cientifica.edu.pe (DWR) \\ ${ }^{5}$ Laboratorio de Florística, Museo de Historia Natural, Universidad Nacional Mayor de San Marcos. Av. Arenales 1256, CP. 15072, \\ Lima. Lima, Perú. \\ ${ }^{*}$ Corresponding author
}

Cavia tschudii inhabits coastal and Andean wetlands where it is important prey for medium carnivores, but its habitat selection and its role in the wetlands are unknown. In order to reduce this lack of knowledge, we evaluated changes in the abundance for two seasons and the habitat selection of $C$. tschudii in a wetland on the central coast of Peru. Additionally, we report information on their movement distances and provide comments on their predators. We carried out six evaluations during the autumn and spring of 2019, with nine grids in three plant communities: grassland, bulrush community, and cattail community. Each captured individual was marked with a numbered ear tag. We compared the relative abundance between seasons using the Mann-Whitney $U$ test, and calculated the relative abundance per evaluated month and carried out regressions to model its behavior. The use/availability of habitat was evaluated with the Chi-square test together with Bonferroni confidence intervals to show habitat selection. Finally, we estimate the mean maximum distance moved (MMDM) of recaptured individuals. The results show significant differences between the seasons, with higher relative abundance in autumn. The relative abundance showed a peak in April, from which the values decrease. Likewise, we found significant differences in habitat selection, showing positive selection for the cattail community, neutral selection for the bulrush community, and negative selection for the grassland. The MMDM was $36.5 \pm 15.7 \mathrm{~m}$. We report a decrease in the relative abundance of $C$. tschudii, possibly related to seasonal changes in habitat quality or to the presence of predators. The peak of abundance in mid-autumn and the apparent decrease in the population until late spring resembles the annual dynamics reported for C. aperea. It is also confirmed that C. tschudii, like other Cavia, select environments with greater plant coverage, possibly as an anti-predation strategy. We report dogs predating $C$. tschudii and provide a list of other potential predators. This study increases the information on C. tschudii in coastal wetlands and gives a first approach to the necessary knowledge for its management and conservation within these fragile ecosystems.

Cavia tschudii habita humedales costeros y andinos donde es presa importante de carnívoros medianos, pero su selección de hábitat y su rol en los humedales son desconocidos. Con el fin de disminuir estos vacíos de información, evaluamos cambios en la abundancia de $C$. tschudii durante dos estaciones del año y su selección de hábitat en un humedal de la costa central del Perú. Adicionalmente, reportamos información sobre sus movimientos y ofrecemos comentarios sobre sus depredadores. Realizamos seis evaluaciones durante el otoño y primavera de 2019 ; con nueve cuadrantes en tres comunidades vegetales: gramadal, juncal y totoral. Cada individuo capturado fue marcado con un arete numerado. Comparamos las abundancias relativas entre estaciones mediante la prueba de U de Mann-Whitney. Además, calculamos las abundancias relativas por mes evaluado y realizamos regresiones para modelar su comportamiento. Evaluamos el uso/disponibilidad del hábitat con la prueba Chi-cuadrado junto con intervalos de confianza de Bonferroni para evidenciar selección de hábitat. Además, estimamos el promedio de distancias máximas de movimiento (MMDM). Los resultados muestran diferencias significativas entre las estaciones, siendo la abundancia relativa mayor en otoño. La abundancia relativa mostró un pico en abril, a partir del cual los valores descienden. Asimismo, encontramos diferencias significativas en la selección de hábitat, mostrando selección positiva por el totoral, selección neutra por el juncal y selección negativa por el gramadal. El MMDM fue de $36.5 \pm 15.7 \mathrm{~m}$. Reportamos una disminución en la abundancia relativa de $C$. tschudii, posiblemente relacionados a cambios estacionales en la calidad de hábitat o en la presencia de depredadores. El pico de abundancia a mediados de otoño y el aparente decrecimiento de la población hasta fines de la primavera, se asemeja a dinámicas anuales reportadas en C. aperea. Confirmamos también que C. tschudii, similar a otros Cavia, prefiere ambientes con mayor cobertura vegetal; posiblemente como una estrategia anti-depredación. Reportamos perros depredando C. tschudii y proveemos una lista de otros depredadores potenciales. Este estudio incrementa la información sobre C. tschudii en humedales costeros y da un primer acercamiento al conocimiento necesario para su manejo y conservación dentro de estos ecosistemas frágiles.

Keywords: Habitat selection; movement distance; Ramsar wetland; relative abundance; small mammal. 


\section{Introducción}

Cavia tschudii es la especie de cuy silvestre que originó al cuy doméstico C. porcellus (Dunnum y Salazar-Bravo 2010). Esta especie se distribuye principalmente en los Andes del Perú, Bolivia, Chile y Argentina, con un rango desde los 0 a 4500 msnm. Habita humedales costeros, andinos y pastizales donde es una importante presa de carnívoros medianos (Dunnum 2015). A nivel internacional, su estado de conservación es Preocupación Menor de acuerdo a la Lista Roja de la Unión Internacional para la Conservación de la Naturaleza (UICN; Dunnum y Teta 2016); sin embargo, el conocimiento sobre las poblaciones de esta especie es escaso. Al límite sur de su distribución, en Argentina y Chile, ha sido categorizado como una especie con Datos Insuficientes (Cirignoli 2019) y Rara (Ministerio de Agricultura 1998, 2015) respectivamente por los escasos registros, desconocimiento general sobre el estado de sus poblaciones y de las amenazas que pueden afectar a las poblaciones que habitan el territorio de cada país. En Bolivia, Tarifa et al. (2010) evidencian la falta de información sobre su ecología; especialmente sobre la abundancia y densidad poblacional de C. tschudii.

En Perú, se conoce su presencia en las ecorregiones de serranía esteparia, puna y desierto costero (Pacheco et al. 2009). En esta última ecorregión, los cuyes silvestres habitan los humedales costeros, ecosistemas que son considerados de acuerdo con la legislación peruana como ecosistemas frágiles de conservación prioritaria (Congreso de la República 2005). A pesar de ello, estos ecosistemas son continuamente afectados principalmente por la agricultura y la ganadería (Aponte y Ramirez 2011), así como por otros procesos antropogénicos como la urbanización, cambio de uso de suelo e incendios (Aponte et al. 2015; Gonzales et al. 2019; Flores et al. 2020). Particularmente en este tipo de ambientes la información es escasa; los estudios publicados solo abordan registros de presencia de la especie (Zeballos 2010; Pacheco et al. 2015), un reporte de amenaza por incendios (Ramirez et al. 2018) y un caso de albinismo (Ramirez et al. 2019).

El Refugio de Vida Silvestre Los Pantanos de Villa (Pantanos de Villa) es un Área Natural Protegida (ANP) amenazada por la creciente presión urbana (SERNANP 2016) y el único humedal costero del departamento de Lima donde se ha reportado la especie (Pacheco et al. 2015). Este humedal es importante por su belleza paisajística, la gran diversidad biológica que alberga y por ser un lugar esencial en el recorrido migratorio para numerosas especies de aves a lo largo de la región Neotropical. Por ello, los estudios en esta ANP se han enfocado principalmente en su vegetación y sus aves (Cano et al. 1993; Wust et al. 1994; Ramirez y Cano 2010; Pulido 2018; Flores et al. 2020).

En este sentido, con el fin de aumentar el conocimiento sobre las poblaciones de $C$. tschudii en los humedales costeros, nos planteamos dos objetivos: evaluar cambios en la abundancia de C. tschudii en el humedal Pantanos de Villa durante otoño y primavera del 2019 y su selección de hábitat dentro de este humedal costero. Adicionalmente, proveemos comentarios sobre depredadores potenciales y confirmados de C. tschudii en el área de estudio y reportamos información sobre el movimiento de sus individuos.

\section{Materiales y métodos}

Área de estudio. Pantanos de Villa se ubica en el departamento y provincia de Lima (Perú), en el distrito de Chorrillos, entre los kilómetros 18 y 21 de la antigua carretera panamericana sur $\left(-12^{\circ} 11^{\prime} 42^{\prime \prime},-12^{\circ} 13^{\prime} 18^{\prime \prime} \mathrm{S},-76^{\circ} 58^{\prime} 42^{\prime \prime},-76^{\circ}\right.$ $59^{\prime} 42^{\prime \prime} \mathrm{W}$; Figure 1). El área se encuentra rodeada de zonas urbanas, clubes campestres, un club hípico y una universidad. El ecosistema comprende una superficie de 263 ha (Ministerio de Agricultura 2006). Debido a que el humedal es un refugio de aves migratorias, fue reconocido internacionalmente a partir del 20 de febrero de 1997 como un humedal de importancia internacional o sitio RAMSAR. En el 2006 fue recategorizado a nivel nacional como Refugio de Vida Silvestre. El ecosistema está conformado por seis espejos de agua de diferentes tamaños (Laguna Mayor, Laguna Génesis, Laguna ANAP, Laguna Marvilla, Laguna Las Garzas y Laguna La Pampa), dos canales principales que abastecen de agua a todo el humedal, un afloramiento y zonas pantanosas con abundante materia orgánica de origen vegetal y terrenos calcáreos-arenosos (Ramirez et al. 2018).

El clima se caracteriza por una estación húmeda durante los meses de julio y septiembre (temperatura media: 15 a $17^{\circ} \mathrm{C}$ ) y una estación seca entre diciembre y abril (temperatura media: 23 a $25^{\circ} \mathrm{C}$ ). La ausencia de lluvias en la costa central peruana es casi constante, oscilando entre 0 y 5.5 $\mathrm{mm}$ de precipitación total mensual promedio (Ministerio del Ambiente 2015; Pulido 2018). Con el fin de comparar este estudio con otros estudios poblacionales del género Cavia, consideramos las estaciones de otoño y primavera, comprendiendo el otoño los meses antes de la época húmeda (de marzo a junio) y la primavera los meses posteriores a la época húmeda (de septiembre a diciembre). El otoño se caracteriza por ser una transición entre la época seca y húmeda, donde las temperaturas medias van descendiendo según avanzan los meses (de los $23^{\circ} \mathrm{C}$ a los $19^{\circ} \mathrm{C}$ ). Por el contrario, en la primavera las temperaturas van aumentando con el avance de los meses (de $17{ }^{\circ} \mathrm{C}$ a $22^{\circ} \mathrm{C}$; CORPAC S. A. 2020; SENAMHI 2020).

La vegetación predominante es de tipo herbácea y está representada por comunidades vegetales como totorales (66.1 ha), juncales (52.2 ha), gramadales (73.4 ha), zonas arbustivas (5.8 ha) y acuáticas (1.8 ha; Cano y Young 1998; Aponte et al. 2018). En el presente estudio evaluamos tres tipos de vegetación: 1) gramadal; vegetación típicamente dominada por Distichlis spicata y menos frecuente por Sporobolus virginicus "grama salada", especies que alcanzan alturas de 50 a $70 \mathrm{~cm}$ y presentan coberturas cercanas al 60 a $80 \%$; en algunos sectores puntuales del humedal el gramadal es dominado por Paspalum vaginatum "grama dulce" con presencia de un suelo más húmedo y terroso (Figure 2a). 2) juncal; comunidad vegetal dominada por Schoenoplectus americanus "junco" que alcanza alturas de 


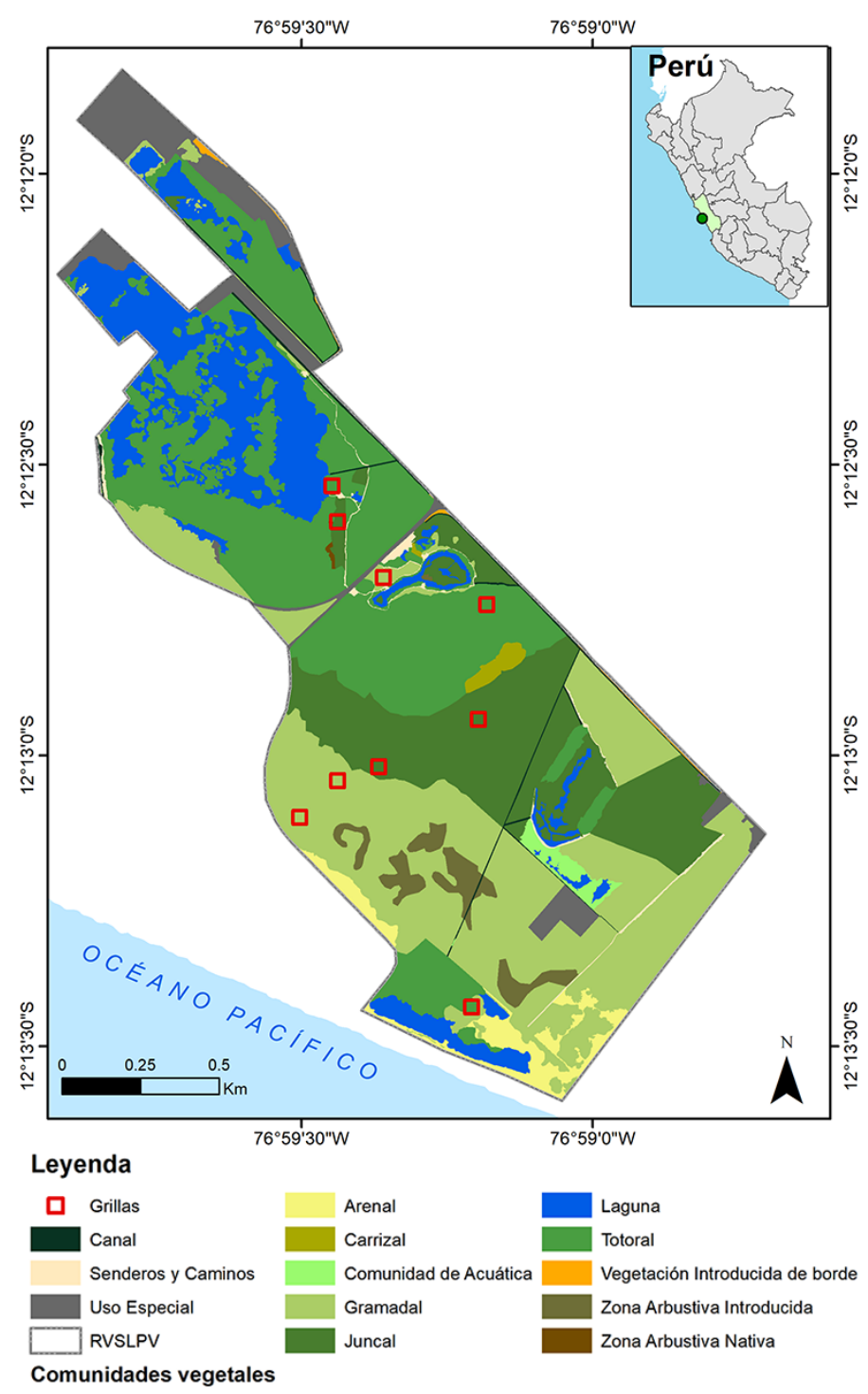

Figura 1. Ubicación del Refugio de Vida Silvestre Los Pantanos de Villa (Lima, Perú), comunidades vegetales que lo componen y distribución de grillas de muestreo dentro del área de estudio.

1.0 a $1.5 \mathrm{~m}$ y presenta coberturas cercanas al $100 \%$; en algunos juncales se puede observar una codominancia con D. spicata; el sustrato donde se desarrolla esta comunidad se encuentra húmedo a saturado y depende de la dinámica del agua subterránea para su distribución en el humedal (Figure 2b). 3) totoral; comunidad vegetal dominada por Typha domingensis "totora" de 2.0 a $2.5 \mathrm{~m}$ de altura, la especie puede cubrir rápidamente los espacios libres en los bordes de los cuerpos de agua y presenta coberturas cercanas al 80 a $100 \%$; el sustrato de esta comunidad se caracteriza por estar siempre saturado o inundado; la vegetación se desarrolla principalmente en los bordes de las lagunas o cubriendo canales de escorrentía lenta (Figure 2c). No evaluamos las zonas arbustivas por su poca extensión y por no hallar indicios de la presencia del cuy silvestre (Aponte et al. 2018; Quispe-López obs. pers.).

Diseño de muestreo. Realizamos evaluaciones mensuales durante seis meses, abarcando el otoño (de marzo a mayo) y la primavera (de septiembre a diciembre) del 2019. En cada evaluación establecimos nueve cuadrantes, colocando tres por cada comunidad vegetal (totoral, juncal, gramadal; Figure 1); excepto por el gramadal en las dos primeras evaluaciones, donde contó sólo con una grilla en la primera evaluación y con dos en la segunda. Los cuadrantes estuvieron activos cinco días continuos por evaluación. Cada cuadrante consistió en un arreglo de $3 \times 4$ trampas Tomahawk abarcando un área de $30 \times 40 \mathrm{~m}^{2}$. Cada trampa fue cebada con una mezcla de hojas de alfalfa y conejina humedecida (alimento balanceado para conejo), y cubierta con la vegetación circundante para reducir el estrés en los individuos capturados.

La distancia entre cuadrantes continuos fue $310 \pm 222 \mathrm{~m}$ (promedio $\pm S D$ ) (distancia mínima 113 m, distancia máxima $804 \mathrm{~m}$ ). Buscamos distanciar los cuadrantes al menos 125 $\mathrm{m}$ por ser la longitud máxima de área de acción registrada para C. aperea (Asher et al. 2004). Sin embargo, por lo relativamente pequeña del área de estudio dos cuadrantes se distanciaron $113 \mathrm{~m}$. Al respecto, utilizando el marcaje de individuos durante este estudio, obtuvimos el valor promedio de distancias máximas de movimiento (mean maximum distance moved, MMDM) y lo consideramos un proxy del área de acción (Wilson y Anderson 1985; Karanth y Nichols 1998); basados en ello, confirmamos que el distanciamiento mínimo entre cuadrantes fue suficiente para este estudio (ver resultados). Calculamos el esfuerzo de muestreo multiplicando el número de trampas activas por el número de noches que las trampas estuvieron activas, obteniendo la cantidad de trampas/noche (T/N; Grinnell 1914; Lim y Pacheco 2016).

Captura deindividuos. Cada individuo capturado fue marcado con un arete de código único (ear tag 1005-1, National Band \& Tag Co., Kentucky, Estados Unidos) y liberado en el mismo punto de captura (Figure 2d), permitiéndonos distinguir las recapturas. El estrés observado en los animales capturados fue reducido cubriendo las trampas antes de cada manipulación. Durante las recapturas no observamos daños causados por los aretes. Los métodos descritos fueron previamente aprobados por la autoridad nacional SERNANP (RJ-RVSLPV No005-2019-SERNANP-JEF), la autoridad municipal PROHVILLA (carta de consentimiento, 04-042019) y por el comité ético de la Universidad Científica del Sur (constancia No 38-CIEI-AB-CIENTIFICA-2019). Asimismo, seguimos los lineamientos de la American Society of Mammalogists para el uso de mamíferos silvestres en investigación y educación (Sikes et al. 2016).

Cambios en la abundancia. Para evaluar cambios en la abundancia de $C$. tschudii comparamos las abundancias relativas entre las estaciones de otoño y primavera. Calculamos las abundancias relativas con el índice de capturabilidad (W; Pucek 1981). Dicho índice estandariza el esfuerzo de muestreo expresando la abundancia relativa como el número de individuos capturados por cada 100 trampas-noche (Pacheco et al. 2007; Salas et al. 2013). Las comparaciones las hicimos por cada comunidad vegetal y agrupando las tres comunidades vegetales; obtuvimos la significancia mediante la prueba estadística U de MannWhitney en el software Minitab 17 (2010). 
Para evaluar los cambios mensuales graficamos y modelamos el comportamiento de la muestra de población que obtuvimos durante el periodo de estudio realizando múltiples regresiones no polinómicas seguidas de un proceso de selección de modelos; las variables utilizadas fueron la abundancia relativa y el tiempo (en meses, se consideró el primer mes de muestreo como el mes 1); para ello, se utilizó el coeficiente de correlación $(r)$, el coeficiente de determinación $\left(R^{2}\right)$ y el Criterio de Información de Akaike (AIC). El mejor modelo fue aquel que tuvo un AIC más bajo (que representa un modelo de mejor calidad) y un $R^{2}$ y $r$ más cercano a la unidad (que nos indica que ese modelo representa mejor la distribución de la data evaluada). Todo este procedimiento fue realizado mediante la función Curve finder del programa Curve Expert Professional 2.6.5 (Hyams 1996).

Selección de hábitat. Consideramos el hábitat como un ambiente delimitado por las asociaciones vegetales (Hall et al. 1997), las capturas como un indicador de uso de hábitat y la disponibilidad como el área de las comunidades vegetales estudiadas. La existencia de selección negativa o rechazo, y selección positiva de $C$. tschudii hacia alguna comunidad vegetal fue evaluada determinando las diferencias entre los valores del uso y la disponibilidad de hábitat con la prueba de Chi-cuadrado de bondad de ajuste donde los datos observados son las capturas por hábitat y los datos esperados son calculados con la multiplicación entre el área relativa del tipo de hábitat y el número total de individuos (Byers et al. 1984). Las áreas de cada comunidad vegetal fueron tomadas del mapa de vegetación del humedal (Aponte et al. 2018). Cuando estas diferencias fueron estadísticamente significativas, se calcularon los intervalos simultáneos de Bonferroni al $95 \%$ de confianza a partir del número de individuos observados y el área relativa de cada comunidad vegetal (Neu et al. 1974; Byers et al. 1984).

Promedio de distancias máximas de movimiento (MMDM). Calculamos el MMDM de C. tschudii para ambas temporadas y por cada temporada por separado. Para ello utilizamos la siguiente fórmula (Williams et al. 2002): $d=\sum_{i=1}^{\mathrm{m}} d_{i} / \mathrm{m}$. Donde $₫=$ Promedio de distancias máximas de movimiento, $m=$ número de individuos capturados al menos dos veces, $d_{i}=$ distancia máxima entre lugares de captura de cada individuo $i$ capturado al menos dos veces.

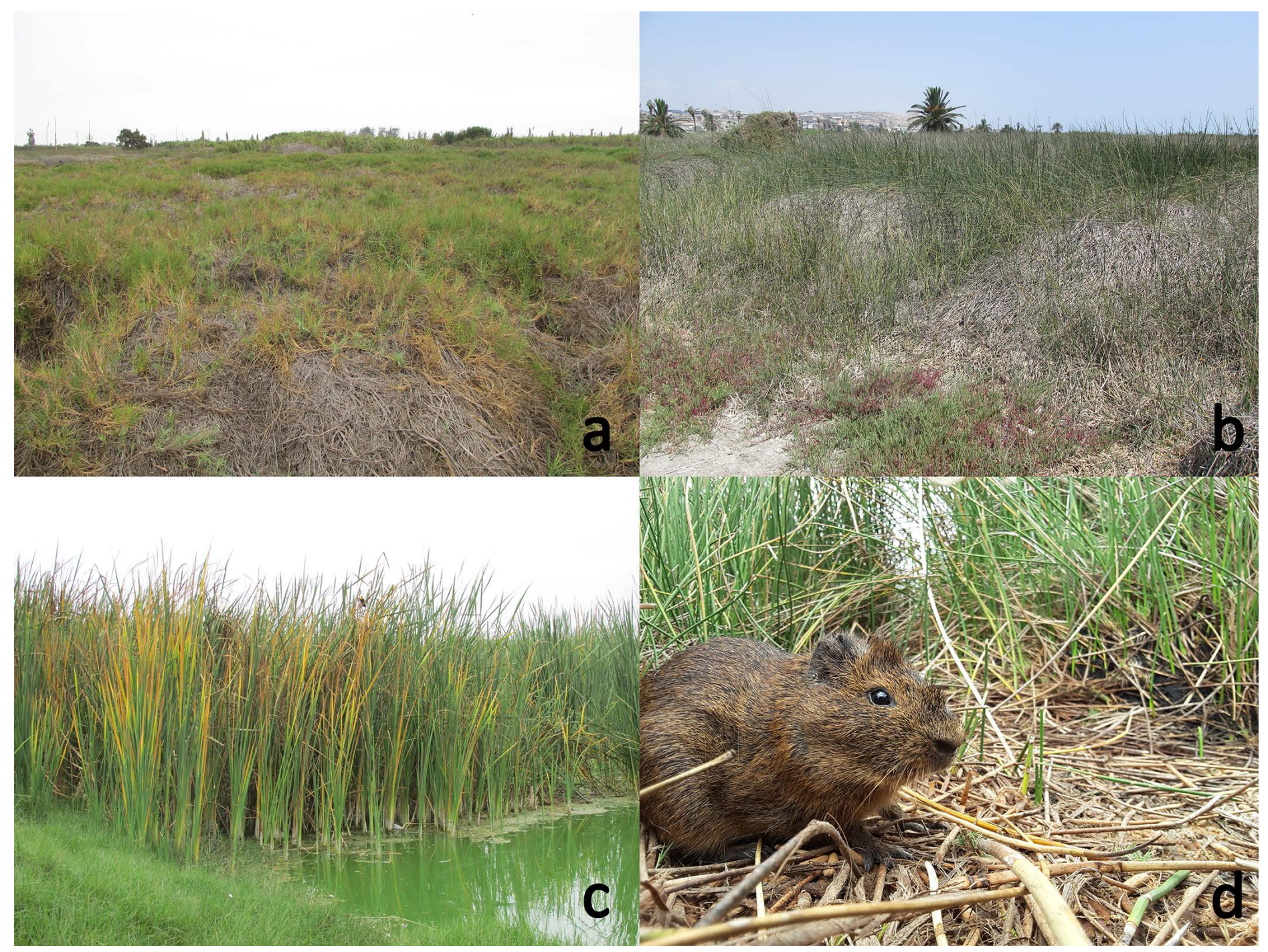

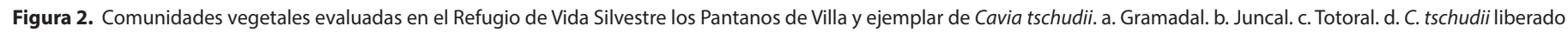
en su punto de captura. 
Comentarios sobre depredadores. Reportamos evidencias de depredación observadas durante el trabajo de campo. Asimismo, revisamos la literatura publicada sobre rapaces presentes en Pantanos de Villa (Pulido 2018) y elaboramos un cuadro (Apéndice 1) señalando los potenciales depredadores de C. tschudii en base a reportes de consumo de especies del género Cavia en otros lugares, o consumo de otros roedores.

\section{Resultados}

Con un esfuerzo de muestreo total de 3,060 trampas/noche ( $\mathrm{T} / \mathrm{N}$ ) obtuvimos 43 capturas de 33 individuos; incluyendo 15 machos, 15 hembras y 3 indeterminados. Veinticinco individuos fueron capturados durante el otoño $(1,440 \mathrm{~T} / \mathrm{N})$ y 11 durante la primavera $(1,620 \mathrm{~T} / \mathrm{N}$; Tabla 1). Seis individuos fueron recapturados a lo largo del estudio, tres fueron recapturados una vez, dos recapturados dos veces, y sólo un individuo fue recapturado tres veces.

Cambios en la abundancia. Al comparar el otoño y la primavera en cada comunidad vegetal las abundancias relativas promedio siempre fueron mayores en otoño (Figure 3). Sin embargo, el test de Mann-Whitney no soportó estas diferencias (gramadal $P=0.11$; juncal $P=0.282$; totoral $P=0.107$ ). Por otro lado, al agrupar los datos de las tres comunidades vegetales y compararlos entre las dos estaciones, se hallaron diferencias significativas $(P<0.01)$ siendo la abundancia relativa mayor en otoño (Figure 3 ).

Durante los meses evaluados registramos un pico de abundancia relativa en abril a partir del cual los valores descendieron (Figure 4). Por otro lado, el proceso de selección de modelos brindó la puntuación más alta a tres modelos: el modelo de DR-Hill, el modelo Morgan-MercerFlodin (MMF), y el modelo de Weibull. Dichos modelos mostraron los mismos valores de $r(0.96), R^{2}(0.93)$ y AIC (-3.34) que les brindan confianza matemática. Las tres ecuaciones muestran la disminución de la población en el período de estudio. Por la menor complejidad de la ecu-

Tabla 1. Número de individuos capturados de Cavia tschudii en tres comunidades vegetales de Pantanos de Villa durante otoño y primavera del 2019.

\begin{tabular}{|c|c|c|c|c|c|c|}
\hline \multirow[b]{2}{*}{ Mes } & \multicolumn{3}{|c|}{ Comunidad vegetal } & \multicolumn{2}{|c|}{ Sexo } & \multirow[b]{2}{*}{ Total } \\
\hline & Gramadal & Juncal & Totoral & 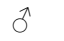 & q & \\
\hline \multicolumn{7}{|l|}{ Otoño } \\
\hline Marzo & 2 & 2 & 5 & 2 & 5 & $9 *$ \\
\hline Abril & 1 & 4 & 6 & 3 & 7 & $11^{*}$ \\
\hline Mayo & 1 & 3 & 4 & 3 & 5 & 8 \\
\hline \multicolumn{7}{|l|}{ Primavera } \\
\hline Septiembre & 0 & 2 & 2 & 2 & 2 & 4 \\
\hline Noviembre & 1 & 2 & 3 & 5 & 1 & 6 \\
\hline Diciembre & 0 & 0 & 3 & 2 & 1 & 3 \\
\hline Total & 5 & 13 & 15 & 15 & 15 & 33 \\
\hline
\end{tabular}

*Contando individuos de sexo indeterminado.

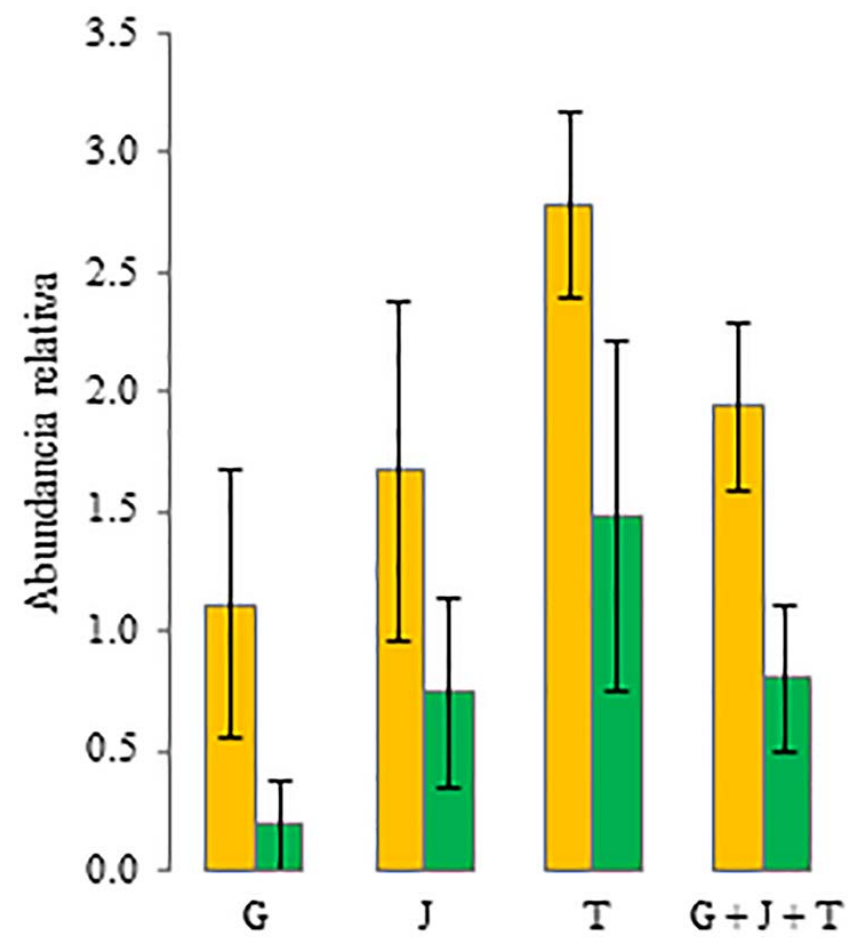

Figura 3. Abundancias relativas (promedio $\pm I C$ ) de Cavia tschudii en Pantanos de Villa durante otoño y primavera del 2019. Comparaciones por cada comunidad vegetal y considerando las tres comunidades juntas. Las abreviaciones son: G, gramadal; J, juncal; T, totoral. Otoño (de marzo a mayo; naranja); Primavera (de septiembre a diciembre, verde).

ación escogimos describir el modelo MMF (Figure 4); la ecuación obtenida es la siguiente: $y=a b+c x^{d} / b+x^{d}$. Donde $x$ $=$ es el tiempo en meses; $y=$ la abundancia relativa; $a=8.02$; $\mathrm{b}=9.35 ; \mathrm{c}=-2.22$ y $\mathrm{d}=-1.9$.

Selección de hábitat. El valor obtenido de Chi-cuadrado $\left(X^{2}\right)$ fue de $14.10(n=43 ; P<0.01 ; g . I .=2)$. Un análisis de los datos obtenidos encuentra que el totoral fue el hábitat más usado de lo esperado, evidenciando selección positiva. Por el contrario, el gramadal fue la comunidad vegetal donde $C$. tschudii tuvo una proporción de uso menor a lo esperado, mostrando una selección negativa. El juncal no presentó diferencias significativas entre su disponibilidad y el uso de los cuyes silvestres (Tabla 2).

Promedio de distancias máximas de movimiento. Considerando ambas temporadas obtuvimos un MMDM de 36.5 $\pm 15.7 \mathrm{~m}$ (promedio $\pm S D$ ), siendo la mayor distancia máxima de movimiento registrados para un mismo individuo de $54.8 \mathrm{~m}$ en los totorales. El MMDM en otoño fue de $27.2 \pm$ $14.9 \mathrm{~m}$ y en primavera $34.8 \pm 8.8 \mathrm{~m}$. La distancia máxima de movimiento de un mismo individuo en otoño fue 43.6 $\mathrm{m}$ y en primavera $44 \mathrm{~m}$. Teniendo en cuenta que ningún individuo capturado en una grilla fue recapturado en otra grilla distinta, confirmamos que el distanciamiento mínimo establecido entre grillas fue suficiente para este estudio.

Comentarios sobre depredadores. Durante el trabajo de campo observamos evidencias que señalarían a los perros como uno de los principales depredadores de cuyes silvestres dentro de Pantanos de Villa. Observamos al menos 10 perros distintos formando grupos que recorrían el área de estudio revisando senderos y madrigueras de $C$. tschudii. 


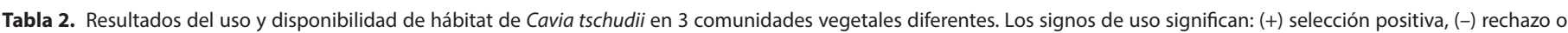
selección negativa y (n.s.) no hay selección.

\begin{tabular}{|c|c|c|c|c|c|c|c|c|}
\hline Comunidad vegetal & Área total (ha) ${ }^{1}$ & Uso observado & Uso esperado & Proporción de uso observado $\left(p_{i}\right)$ & Proporción de uso esperado & \multicolumn{3}{|c|}{ Intervalos de Bonferroni } \\
\hline Gramadal & 73.4 & 5 & 16 & 0.116 & $0.383(-)$ & 0.00 & $\leq \mathrm{p}_{\mathrm{i}} \leq$ & 0.23 \\
\hline Juncal & 52.2 & 14 & 11 & 0.326 & 0.272 (n.s.) & 0.16 & $\leq \mathrm{p}_{\mathrm{i}} \leq$ & 0.50 \\
\hline Totoral & 66.12 & 24 & 15 & 0.558 & $0.345(+)$ & 0.38 & $\leq \mathrm{p}_{\mathrm{i}} \leq$ & 0.74 \\
\hline
\end{tabular}

'Tomado de Aponte et al. (2018).

Asimismo, dentro de los totorales hallamos un refugio con cachorros de perros y carcasas de cuyes silvestres; y en una ocasión pudimos observar directamente perros cazando un cuy silvestre.

Entre las rapaces presentes resaltan Tyto alba y Parabuteo unicinctus por ser residentes de la zona y tener reportes donde especies del género Cavia han sido la presa principal en cuanto a biomasa consumida (Vargas et al. 2002; AliagaRossel y Tarifa 2005; Salvador 2012; Gómez y Lires 2015).

\section{Discusión}

Cambios en la abundancia. Nuestros resultados muestran una disminución en la población de $C$. tshudii a nivel de las tres comunidades vegetales juntas, donde la mayor abundancia fue durante el otoño. Al no haber otros estudios sobre variación en poblaciones de $C$. tschudii, no es posible complementar estos resultados para la especie. No obstante, existen estudios de este tipo en otras especies del mismo género las cuales muestran que algunas de sus especies siguen un patrón estacional. Galante y Cassini (1994) y Bilenca et al. (1995) encontraron fluctuaciones estacionales en las densidades de poblaciones de C. aperea en Argentina. Los picos de densidad más altos ocurrieron a finales de otoño e inicios de invierno, declinaron durante el invierno y alcanzaron sus niveles más bajos en primavera o verano; a partir del cual, los valores de densidad volvieron a incrementar hasta llegar a un nuevo pico en otoño. Nuestros resultados sugieren un patrón parecido: la abundancia relativa tuvo sus valores más altos durante el otoño (marzo y abril), empezó a descender a finales de esta temporada (mayo), y alcanzaría sus valores más bajos durante el invierno y primavera (de junio a diciembre; Figure 4). De cumplirse este patrón, las abundancias relativas empezarían a aumentar durante el verano, alcanzando nuevamente sus valores más altos durante el otoño. Sin embargo, para poder confirmar un patrón estacional, son necesarios estudios que abarquen todo el año durante más años.

Las dinámicas estacionales en especies del género Cavia son principalmente atribuidas a la disminución de la calidad de hábitat (Galante y Cassini 1994; Bilenca et al. 1995) o a la presión por depredación (Kraus y Rödel 2004). Respecto a la disminución de calidad de hábitat, las especies vegetales dominantes en humedales como Pantanos de Villa sufren una disminución de biomasa durante las épocas secas (Mitsch et al. 2009; López-Rosas y Moreno-Casasola 2012). Este cambio puede interpretarse como una disminución en la calidad de hábitat para C. tschudii pues disminuiría la calidad de alimento y la cobertura vegetal que los protege de depredadores, incrementando la mortalidad y disminuyendo el número de individuos. Por otro lado, respecto a cambios en la presión por depredación, se ha reportado que $C$. magna presenta estacionalidad en su abundancia debido a cambios estacionales en la presencia de Galictis cuja (Kraus y Rödel 2004). Una dinámica parecida podría estar ocurriendo en Pantanos de Villa, donde algunas de las aves rapaces siguen hábitos migratorios (Pulido y Bermúdez 2018). El cambio estacional en la presencia de estas aves rapaces podría propiciar cambios estacionales en la abundancia de $C$. tschudii.

Selección de hábitat. Este trabajo es el primer estudio realizado sobre selección de hábitat de $C$. tschudii, por lo que resulta complejo mostrar semejanzas con otras investigaciones. Sin embargo, muchos estudios muestran que otras especies del género Cavia también seleccionan hábitats caracterizados por su densa cobertura. Asher et al. (2004) evidenciaron selección positiva de C. aperea para una vegetación densa de largas cañas de más de $3 \mathrm{~m}$ de longitud, crecidas en áreas húmedas como las orillas de un lago o pequeños arroyos en Brasil. En Argentina, Guichón y Cassini (1998) no encontraron diferencias significativas sobre el uso de hábitat por $C$. aperea respecto a la disponibilidad de plantas que consumen, pero sí a los ambientes con cobertura vegetal de más de $1.5 \mathrm{~m}$ de longitud. Asimismo, las otras especies de roedores que habitan Pantanos de Villa, también fueron más abundantes en los totorales, aunque no se evaluó selección de hábitat (Pacheco et al. 2015). Este patrón de comportamiento podría deberse a una estrategia anti-depredación. Ebensperger y Hurtado (2005) explican que el animal que selecciona un ambiente con cobertura vertical busca protección frente a los depredadores aéreos a costa de su visibilidad lateral, facilidades en el escape y la detección de depredadores terrestres.

La selección negativa de C. tschudii por los gramadales, puede deberse también a la presencia de potenciales depredadores. Pulido et al. (2020) analizaron la selección de hábitat de las aves residentes y migratorias del ANP, evidenciando el siguiente comportamiento de las aves rapaces: todas las especies de la familia Accipitridae y de las ordenes Falconiformes y Strigiformes estaban presentes en los gramadales; todos los falcónidos y cinco de los seis accipitriformes fueron encontrados en la vega de ciperáceas (entendida como juncales para el presente estudio) y no hubo registros de ningún falcónido, accipitriforme o strigiforme en los totorales. Por otro lado, durante el trabajo en campo observamos evidencias de depredación de cuyes silvestres por parte de perros en los tres hábitats eval- 
uados, siendo más recurrente su presencia en los gramadales. Amaro y Goyoneche (2017) atribuyeron que la disminución de nidos de aves en Pantanos de Villa se debe a la presencia de especies introducidas como perros, caballos y actividades humanas. Sin embargo, se necesitan estudios que evidencien dicho impacto en la selección de hábitat y población del cuy silvestre.

Dentro de losjuncalesy gramadales, hemos observado que C. tschudii mostraría otras estrategias para disminuir el riesgo de exposición a depredadores como el uso de madrigueras, las cuales no fueron vistas en los totorales. Asimismo, en el humedal de Caucato (Ica, Perú), se ha observado a C. tschudii realizar actividades de forrajeo en grupo a cortas distancias desde la zona con mayor cobertura vegetal (V. Pacheco obs. pers.). Ambos comportamientos también fueron reportados en C. aperea (Asher et al. 2004; Cassini 1991).

Desde el punto de vista de la conservación, se destaca que C. tschudii muestre un mayor uso de hábitat en totorales y juncales, pues ambas comunidades vegetales se han visto afectadas por amenazas como la fragmentación de su hábitat e incendios antrópicos (Pulido 2018; Ramirez et al. 2018). En este contexto, recomendamos fortalecer el plan de manejo de estas comunidades vegetales para evitar su degradación, pues probablemente son hábitats fuente y de gran calidad para $C$. tschudii. Adicionalmente, es resaltante que el hábitat seleccionado por C. tschudii sea un ambiente con suelos frecuentemente saturados de agua o inundados. A la fecha no hay estudios que documenten alguna adaptación de $C$. tschudii que le permita minimizar la pérdida de calor al mojarse, un problema al que son susceptibles los mamíferos pequeños por su limitada capacidad de termorregulación (Hull 1973).

Promedio de distancias máximas de movimiento. Al no haber reportes del MMDM para otros Cavia no fue posible comparar adecuadamente el valor obtenido en este estudio para C. tschudii, aun así, mencionaremos algunos estudios que reportan información de movimiento en Cavia. Rood (1972) reporta para C. aperea el promedio de longitud de rango observado (la distancia entre los cuadrantes más exteriores en los que fue observado un mismo individuo), alcanzando $48 \pm 7 \mathrm{~m}$ para machos y $47 \pm 8 \mathrm{~m}$ para hembras. Dicho valor es cercano a lo registrado en este estudio, sin embargo, por corresponder a una metodología distinta, no se puede señalar si el movimiento de $C$. aperea fue mayor o menor que el de $C$. tschudii. En estudios con telemetría, Asher (2004) reporta áreas de acción para C. aperea con distancias máximas de alrededor de $125 \mathrm{~m}$, siendo mucho mayor a lo reportado aquí para C. tschudii; sin embargo, estos $C$. aperea habitaban parches de vegetación alargados que bordeaban un lago, explicando la gran diferencia de valores. Es posible que los C. tschudii en Pantanos de Villa tengan áreas de acción más circulares debido a lo extenso de las comunidades vegetales que habitan. Por otro lado, el MMDM y la distancia máxima de movimiento fueron mayores en primavera, esto podría estar relacionado con una mayor escasez de recursos, correspondiendo también con el descenso de la abundancia reportado en este estudio. Sin embargo, son necesarios estudios de telemetría para poder esclarecer los distintos aspectos de sus áreas de acción.

Comentarios sobre depredadores. Los perros observados durante el estudio probablemente se traten de individuos cimarrones debido a la presencia de cachorros dentro del área natural (Silva et al. 2018). Kraus y Rödel (2004) sostienen que los principales depredadores de C. magna son otros mamíferos. Del mismo modo, los perros podrían estar ocupando este rol con C. tschudii en nuestra área de estudio, pues dentro del área no se han reportado mamíferos silvestres que los puedan depredar (Pacheco et al. 2015).

En este estudio encontramos una disminución en la abundancia relativa de $C$. tschudii durante el periodo de evaluación y sugerimos que este cambio puede ser parte de una dinámica estacional donde la abundancia estaría respondiendo a cambios estacionales de la calidad de hábitat o de la presión por depredación, como ha sido observado en otras especies del género Cavia. Sin embargo, son necesarios estudios a largo plazo para entender la dinámica de la población, pues también pueden ser ciclos normales de la especie. Por otro lado, identificamos una selección positiva por los ambientes con mayor cobertura vegetal y altura como los totorales, mientras que en ambientes menos protectores como los gramadales y juncales $C$. tschudii podría estar usando otras estrategias anti-depredación como el uso de madrigueras y actividades de forrajeo en grupo. Asimismo, reportamos perros, probablemente cimarrones, como depredadores de C. tschudii y presentamos una lista de aves rapaces como potenciales depredadores de esta especie. En este sentido, este estudio contribuye a la escasa información actual sobre $C$. tschudii, brindando un primer acercamiento al conocimiento necesario para el manejo y conservación de esta especie dentro de ecosistemas frágiles como los humedales costeros.

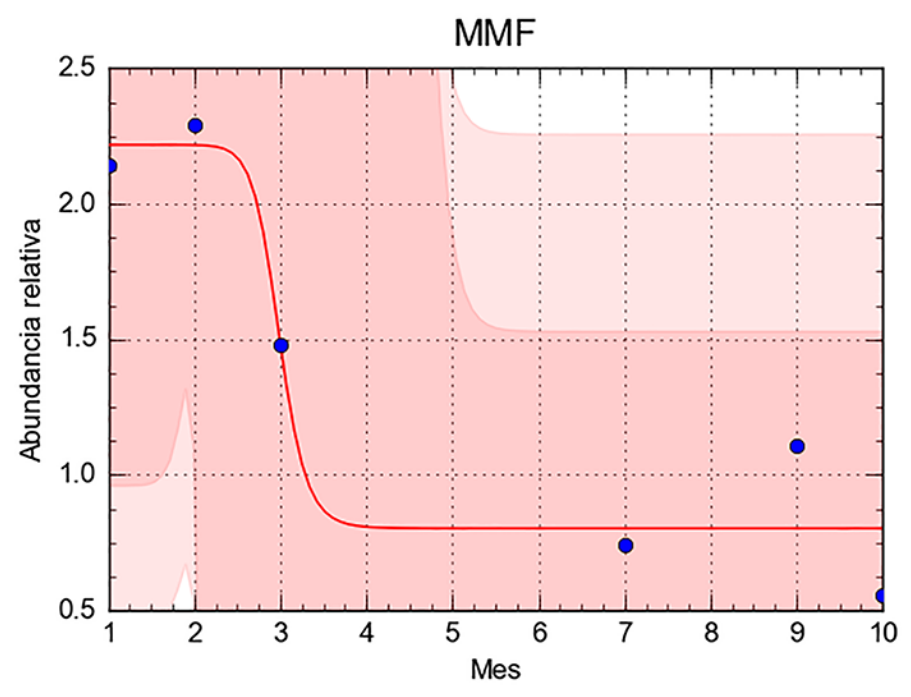

Figura 4. Modelo de regresión Morgan-Mercer-Flodin (MMF) ajustado a la abundancia relativa de Cavia tschudii por mes evaluado durante el 2019 en Pantanos de Villa (mes 1: marzo; mes 10: diciembre; meses 4, 5, 6 y 8 no evaluados). 


\section{Agradecimientos}

El presente estudio se realizó gracias al financiamiento de la Dirección General de Investigación, Desarrollo e Innovación de la Universidad Científica del Sur (código de proyecto: 009-2019-PRO99) con quienes estamos muy agradecidos. Así mismo, agradecemos a la Jefatura del Refugio de Vida Silvestre Los Pantanos de Villa del SERNANP y a la Autoridad Municipal de los Pantanos de Villa - PROHVILLA por las autorizaciones brindadas para la presente investigación, así como a los directores y guardaparques de cada institución quienes nos brindaron las facilidades logísticas durante el trabajo de campo. También agradecemos por el apoyo en el trabajo de campo a B. Mamani, G. Burneo y O. Ruelas, así como al Departamento de Mastozoología del Museo de Historia Natural de la Universidad Nacional Mayor de San Marcos, los estudiantes voluntarios de la Universidad Científica del Sur, al Círculo de Investigación en Ecología de Micromamíferos de la Universidad Nacional Agraria La Molina y a la División de Mastozoología del Centro de Ornitología y Biodiversidad. Damos agradecimientos especiales a los revisores, quienes ayudaron a enriquecer y mejorar las versiones previas de este manuscrito. Finalmente, agradecemos a los señores Honorato y Gustavo Quispe quienes gentilmente nos recibieron en su hogar durante la fase de campo de esta investigación.

\section{Literatura citada}

Aliaga-Rossel, E., y T. Tarifa. 2005. Cavia sp. como principal presa de la lechuza de campanario (Tyto alba) al final de la estación seca en una zona intervenida al norte del Departamento de La Paz, Bolivia. Ecología en Bolivia 40:35-42.

Amaro, L., y G. Goyoneche. 2017. Anidación de aves en el Refugio de Vida Silvestre los Pantanos de Villa 2007-2009, LimaPerú. The Biologist 15:155-171.

Aponte, H., y D. W. Ramirez. 2011. Humedales de la costa central del Perú: Estructura y amenazas de sus comunidades vegetales. Ecología Aplicada 10:31-39.

Aponte, H., D. W. Ramirez, G. Lértora, y F. Gil. 2018. Mapa de cobertura vegetal del Refugio de Vida Silvestre Los Pantanos de Villa. Mapa temático no publicado. Disponible en haponte@cientifica.edu.pe.

Aponte, H., D. W. Ramirez, G. Lértora, R. Vargas, F. Gil, N. CaraZAS, Y R. LIVIAC. 2015. Incendios en los humedales de la Costa Central del Perú: Una amenaza frecuente. Científica 12:70-81.

Asher, M., E. S. De Oliveira, y N. Sachser. 2004. Social system and spatial organization of wild guinea pigs (Cavia aperea) in a natural population. Journal of Mammalogy 85:788-796.

Baladrón, A. V., M. S. Bó, M. Cavalli, y G. Martínez. 2012. Comparación de la dieta de dos especies de rapaces ornitófagas, el halcón perdiguero (Falco femoralis) y el vari (Circus cinereus), en la región pampeana de Argentina. Boletín Chileno de Ornitología 18: 62-67.

Bilenca, D. N., E. A. Cittadino, y F. O. Kravetz. 1995. Influencia de la actividad de Cavia aperea sobre la estructura del hábitat y la distribución de Akodon azarae y Oryzomys flavescens (Rodentia: Caviidae, Muridae) en bordes de cultivos de la región Pampeana (Argentina). Iheringia Serie Zoologia 79: 67-75.
Bó, M., A. Baladrón, y L. Biondi. 2007. Ecología trófica de Falconiformes y Strigiformes: Tiempo de síntesis. Hornero 22:97-115.

Byers, C. R., R. K. Steinhorst, y P. R. Krausman. 1984. Clarification of a technique for analysis of utilization-availability data. The Journal of Wildlife Management 48:1050-1053.

Cadena-Ortiz, H., J. Freille, y D. Bahamonde-Vinueza. 2013. Información sobre la dieta de algunos búhos (Strigidae) del Ecuador. Ornitología Tropical 24:469-474.

Cano A., B. León, y K. Young. 1993. Plantas vasculares de los Pantanos de Villa., Lima. Pp. 177-207, in Las plantas acuáticas en las aguas continentales del Perú (Kahn, F., B. León, y K. Young, comps.). Serie Travaux de l'Institut Français d'Études Andines Tomo 75, Instituto Francés de Estudios Andinos. Lima, Perú.

Cano, A., y K. Young (eds.). 1998. Los Pantanos de Villa: Biología y Conservación. Serie de Divulgación N. ${ }^{\circ} 11$, Museo de Historia Natural, Universidad Nacional Mayor de San Marcos. Lima, Perú.

CASSINI, M. H. 1991. Foraging under predation risk in the wild guinea pig Cavia aperea. Oikos 62:20-24.

CAssinI, M. H., Y M. L. Galante. 1992. Foraging under predation risk in the wild guinea pig: The effect of vegetation height on habitat utilization. Annales Zoologici Fennici 29:285-290.

CIRIGnol, S. 2019. Cavia tschudii. En: Categorización 2019 de los mamíferos de Argentina según su riesgo de extinción. Lista roja de los mamíferos de Argentina (Secretaría de Ambiente y Desarrollo Sustentable de la Nación y Sociedad Argentina para el Estudio de los Mamíferos, eds.). cma.sarem.org.ar. Consultado el 7 de junio 2020.

Congreso de la República. 2005. Ley №28611, Ley general del ambiente. Diario Oficial El Peruano. Perú. 15 de octubre de 2005.

CORPAC S. A. 2020. Tablas y resúmenes climáticos de aeródromos. En: http://www.corpac.gob.pe/app/Meteorologia/TRClimatologicas/Tablas.html. Consultado el 5 de noviembre 2020.

De La Ossa, V., A. De La Ossa-Lacayo, y V. Montes. 2018. Anotaciones etológicas de Mivalgo chimachima, Vieillot, 1816 (Aves: Falconidae). Revista MVZ Córdoba 23:6514-6522.

Dunnum, J. L. 2015. Family Caviidae Fisher, 1817. Pp. 690-726, in Mammals of South America Volume 2: Rodents (Patton, J. L., U. F. J. Pardiñas, y G. D'Elía, eds.). The University of Chicago Press. Chicago, EE.UU.

Dunnum, J. L., y J. Salazar-Bravo. 2010. Molecular systematics, taxonomy and biogeography of the genus Cavia (Rodentia:Caviidae). Journal of Zoological Systematics and Evolutionary Research 48:376-388.

Dunnum, J. L., Y P. TetA. 2016. Cavia tschudii. The IUCN Red List of Threatened Species. Versión 2016.3.1. www.iucnredlist. org. Consultado el 7 de junio 2020.

Ebensperger, L. A., y M. J. Hurtado. 2005. On the relationship between herbaceous cover and vigilance activity of degus (Octodon degus). Ethology 111:593-608.

FiтcH, H. 1974. Observations on the food and nesting of the broad-winged hawk (Buteo platypterus) in northeastern Kansas. The Condor 76:331-360.

Flores, N., I. Castro, y H. Aponte. 2020. Evaluación de las unidades de vegetación en Los Pantanos De Villa (Lima, Perú) mediante sistemas de información geográfica y teledetección. Arnaldoa 27:303-321. 
Galante, M. L., y M. H. Cassini. 1994. Seasonal Variation of a Cavy population in the Pampa region, east-central Argentina. Mammalia 58:549-556.

Gómez, R. O. ,y A. I. LiRes. 2015. Dieta del gavilán mixto (Parabuteo unicinctus) en un humedal de la ciudad de Buenos Aires. Nuestras Aves 60:97-101.

Gonzales, S., H. Aponte, y A. Cano. 2019. Actualización de la flora vascular del humedal Santa Rosa - Chancay (Lima, Perú). Arnaldoa 26:867-882.

GrinnelL, J. 1914. An account of the mammals and birds of the lower Colorado Valley. Zoology 12:51-294.

Guichón, M. L., y M. H. CAssını. 1998. Role of diet selection in the use of habitat by pampas cavies Cavia aperea pamparum (Mammalia, Rodentia). Mammalia 62:23-36.

Hall, L. S., P. R. Krausman, y M. L. Morris. 1997. The habitat concept and a plea for standard terminology. Wildlife Society Bulletin 25:173-182.

HuLl, D. 1973. Thermoregulation in young mammals. Pp. 167200, in Comparative physiology of thermoregulation Volume III (Causey Whittow, G., ed.). Academic Press. New York, EE.UU.

Hyams, D. 1996. CurveExpert: A Curve Fitting System for Windows. Clensom University. Clensom, EE.UU.

Idoeta, F., E I. Roesler. 2012. Presas consumidas por el carancho (Caracara plancus) durante el periodo reproductivo, en el noroeste de la provincia de Buenos Aires. Nuestras Aves 57:80-83.

JAKSIC, F., y J. Simonettı. 1987. Predator/prey relationships among terrestrial vertebrates: an exhaustive review of studies conducted in southern South America. Revista chilena de Historia Natural 60:221-244.

Karanth, K. U., y J. D. Nichols. 1998. Estimation of tiger densities in India using photographic captures and recaptures. Ecology 79:2852-2862.

Kraus, C., y H. G. Rödel. 2004. Where have all the cavies gone? Causes and consequences of predation by the minor grison on a wild cavy population. Oikos 105:489-500.

Lım, B. K., Y V. Pacheco. 2016. Small non-volant mammals. Pp. 83-92 en Core standardized methods for rapid biological field assessment (Larsen, T. H., ed.). Conservation International. Arlington, EE.UU.

López-Rosas, H., y Moreno-Casasola, P. 2012. Invader versus native: Effects of hydroperiod on competition between hydrophytes in a tropical freshwater marsh. Basic and Applied Ecology 13:40-49.

MAder, W. 1982. Ecology and breeding habits of the Savanna Hawk in the Llanos of Venezuela. The Condor 84:261-271.

Ministerio de Agricultura. 1998. Decreto Supremo № 05, Reglamento de la Ley de Caza. Diario Oficial de la República de Chile. Chile. 9 de enero de 1998.

Ministerio de Agricultura. 2006. Decreto Supremo Nº55-2006AG, Que dispone la categorización de la Zona Reservada Los Pantanos de Villa. Gaceta Jurídica, Boletín oficial de normas legales de El Peruano. Perú. Viernes 1 de setiembre de 2006.

Ministerio de Agricultura. 2015. Decreto N65, Modificación al reglamento de la Ley de Caza. Diario Oficial de la República de Chile. Chile. 31 de enero de 2015.

Ministerio del Ambiente. 2015. Guía de inventario de la fauna silvestre. Dirección General de Evaluación, Valoración y Financiamiento del Patrimonio Natural. Lima, Perú.
Minitab 17 Statistical Software. 2010. State College, PA: Minitab, Inc. www.minitab.com

Mitsch, W. J., G. Gosselink, C. Anderson, y L. Zhang. 2009. Wetland ecosystems. John Wiley and Sons Inc. Nueva York, EE.UU.

Neu, C. W., C. R. Byers, y J. M. Peek. 1974. A technique for analysis of utilization-availability data. The Journal of Wildlife Management 38:541-545.

Pacheco, V., R. Cadenillas, E. Salas, C. Tello, y H. Zeballos. 2009. Diversidad y endemismo de los mamíferos del Perú. Revista peruana de biología 16:5-32.

Pacheco, V., E. Salas, L. Cairampoma, M. Noblecilla, H. Quintana, F. Ortiz, P. Palermo, y R. Ledesma. 2007. Contribución al conocimiento de la diversidad y conservación de los mamíferos en la cuenca del río Apurímac, Perú. Revista Peruana de Biología 14:169-180.

Pacheco, V., A. Zevallos, K. Cervantes, J. Pacheco, y J. Salvador. 2015. Mamíferos del Refugio de Vida Silvestre Los Pantanos de Villa, Lima-Perú. Científica 12:26-41.

Pinto, M., H. ZuñIgA, y O. Torres. 2002. Estudio sistemático del género Cavia Pallas, 1766 (Rodentia: Caviidae) en Colombia. Academia Colombiana de Ciencias Exactas, Físicas y Naturales. Bogotá, Colombia.

Pucek, Z. 1981. Keys to vertebrates of Poland: mammals. Polish Scientific Publishers. Varsovia, Polonia.

Pulido, V. 2018. Ciento quince años de registros de aves en Pantanos de Villa. Revista Peruana de Biología 25:291-306.

Pulido, V., y L. Bermúdez. 2018. Patrones de estacionalidad de las especies de aves residentes y migratorias de los Pantanos de Villa, Lima, Perú. Arnaldoa 25:1107-1128.

Pulido, V., Salinas, L., del Pino, J., y Arana, C. 2020. Preferencia de hábitats y estacionalidad de las especies de aves de los Pantanos de Villa en Lima, Perú. Revista Peruana de Biología 27:349-360.

Ramirez, D. W., H. Aponte, G. Lertora, y F. Gil. 2018. Incendios en el humedal Ramsar Los Pantanos de Villa (Lima-Perú): Avances en su conocimiento y perspectivas futuras. Revista de Investigaciones Altoandinas 20:347-360.

Ramirez, D. W., y A. Cano. 2010. Estado de la diversidad de la flora vascular de los Pantanos de Villa (Lima - Perú). Revista Peruana de Biología 17:111-114.

Ramirez, D. W., M. Quispe-López, D. Marcelo-Carranza, y V. PaCHECO. 2019. Primer reporte de albinismo para el cuy silvestre Cavia tschudii (Mammalia: Rodentia). Revista Peruana de Biología 26: 521-524.

Rood, J. P. 1972. Ecological and Behavioural Comparisons of Three Genera of Argentine Cavies. Animal Behaviour Monographs 5:1-83.

Salas, E., C. Barriga, E. Rengifo, y V. Pacheco. 2013. Assessment of the Impact of the PERU LNG Pipeline on Sigmodontine Rodent Populations in a Montane Forest of Ayacucho, Peru. Pp. 101-109, in Monitoring Biodiversity, lessons from a Trans-Andean Megaproject (Alonso, A., F. Dallmeier, y S. P. Servat, eds.). Smithsonian Institution Scholarly Press. Washington, EE.UU.

Salvador, S. A. 2012. Dieta del gavilán mixto (Parabuteo unicinctus) en Villa María, Córdoba, Argentina. Nuestras Aves 57:21-23.

Sánchez, K., A. Malizia, y M. Bó. 2008. Trophic ecology of the burrowing owl (Athene cunicularia) in urban environments of Mar Chiquita Biosphere Reserve (Buenos Aires province, Argentina). Ornitología Neotropical 19:71-80. 
Senamhi (Servicio Nacional de Meteorología e Hidrología del Perú). 2020. Mapa Climático del Perú. En: https://www.senamhi.gob.pe/?p=mapa-climatico-del-peru. Consultado el 5 de noviembre 2020.

Sernanp (Servicio Nacional de Áreas Naturales Protegidas por el Estado). 2016. Plan Maestro del Refugio de Vida Silvestre los Pantanos de Villa 2016-2020. Lima, Perú.

Sikes, R. and The Animal Care and Use Committee of the American Society of Mammalogists. 2016. Guidelines of the American Society of Mammalogists for the use of wild mammals in research and education. Journal of Mammalogy 97:663-688.

Silva, K. V., C. F. Kenup, C. Kreischer, F. A. S. Fernandez, y A. S. PIREs. 2018. Who let the dogs out? Occurrence, population size and daily activity of domestic dogs in an urban Atlantic Forest reserve. Perspectives in Ecology and Conservation 16:228-233.

Tarifa, T., E. Yensen, P. De la Torre, Z. Porcel, y A. Noss. 2010. Caviidae. Pp. 763-804 en Distribución, ecología y conservación de los mamíferos medianos y grandes de Bolivia (Wallace, R. B., H. Gómez, Z. R. Porcel, y D. I. Rumiz, eds.). Centro de Ecología Simon I. Patiño. Santa Cruz, Bolivia.

Vargas, J., C. A. Landaeta, y J. A. Simonettı. 2002. Bats as prey of barn owls (Tyto alba) in a tropical savanna in Bolivia. Journal of Raptor Research 36:146-147.

Williams, B. K., J. D. Nichols, y M. J. Conroy. 2002. Analysis and management of animal populations. Academic Press. San Diego, EE.UU.

Wilson, K. R. y D. R. Anderson. 1985. Evaluation of two density estimators of small mammal population size. Journal of Mammalogy. 66:13-21

Wust, W., A. Luscombe, y T. Valqui. 1994. Las aves de los Pantanos de Villa y alrededores. Asociación de Ecología y Conservación (ECCO). Lima, Perú.

Zeballos, H. 2010. Notas sobre los mamíferos registrados en las lagunas de Mejía entre el año 1996 al 2000. Revista de Investigación Dilloniana 6:46.

Associated editor: Monica Díaz

Submitted: November 11, 2020; Reviewed: December 10, 2020;

Accepted:March 23, 2021;Published on line:April 7, 2021. 


\section{Apéndice 1}

Lista de depredadores potenciales de Cavia tschudii presentes en los Pantanos de Villa y el tipo de presa reportada en la literatura.

\begin{tabular}{|c|c|c|c|}
\hline & C. tschudii & Cavia spp. & Otros roedores \\
\hline Canis lupus familiaris & Este estudio & & \\
\hline Asio flammeus & & Jaksic y Simontetti 1987 & \\
\hline Athene cunicularia & & Sánchez et al. 2008 & \\
\hline Buteo platypterus & & & Fitch 1974 \\
\hline Buteo polyosoma & & & Bó et al. 2007 \\
\hline Buteogallus meridionalis & & & Mader 1982 \\
\hline Caracara plancus & & Idoeta y Roesler 2012 & \\
\hline Circus cinereus & & Baladrón et al. 2012 & \\
\hline Falco femoralis & & & Bó et al. 2007 \\
\hline Falco peregrinus & & & Bó et al. 2007 \\
\hline Falco sparverius & & & Bó et al. 2007 \\
\hline Geranoaetus melanoleucus & & Pinto et al. 2002 & \\
\hline Glaucidium peruanum & & & Cadena-Ortiz et al. 2013 \\
\hline Milvago chimachima & & & De La Ossa et al. 2018 \\
\hline Parabuteo unicinctus & & Gómez y Lires 2015 & \\
\hline Tyto alba & & Aliaga-Rossel y Tarifa 2005 & \\
\hline
\end{tabular}


